\title{
Systematic Policy Analysis for High-assurance Services in SELinux
}

\author{
Gail-Joon $\mathrm{Ahn}^{\dagger}$ and Wenjuan $\mathrm{Xu}{ }^{\dagger}$ \\ University of North Carolina at Charlotte \\ \{gahn,wxu2\}@uncc.edu
}

\author{
Xinwen Zhang \\ Samsung Information Systems America \\ xinwen.z@samsung.com
}

\begin{abstract}
Identifying and protecting the trusted computing base (TCB) of a system is an important task to provide highassurance services since a set of trusted subjects should be legitimately articulated for target applications. In this paper, we present a formal policy analysis framework to identify TCB with the consideration of specific security goals. We also attempt to model information flows between domains in SELinux policies and detect security violations among information flows using Colored Petri Nets.
\end{abstract}

\section{Introduction}

Determining whether a system can be trusted or not is a critical problem in systems and network management. Particularly for security reasons, a local or remote system administrator typically needs to verify if a system meets information security objectives, such as integrity, confidentiality, and availability requirements. For example, in order to deploy applications in distributed and collaborative computing environments, one machine may need to check if another machine currently runs a known good version of an application software on a well-configured, trusted operating system. Otherwise, a remote machine may run buggy or malicious application codes, or may be improperly configured such that the trusted application can be corrupted by untrustful programs or users. Supporting such an important assurance feature with comprehensive security analysis is necessary to trust a target system based on its current security configurations and policies.

Information flow control is the foundation of many security requirements such as integrity and confidentiality. The earliest information flow analysis works adopted a lattice model to illustrate the flow relationship between objects [9]. Those works assume that every object is labelled with a security attribute, and check information flow by examining

†The work of Gail-Joon Ahn and Wenjuan Xu was partially supported by the grants from National Science Foundation (NSF-IIS-0242393) and Department of Energy Early Career Principal Investigator Award (DEFG02-03ER25565). the security labels of the objects in a flow path. Particularly, the lattice model requires that information cannot flow from low integrity objects to high integrity objects. In practical systems, however, under various circumstances, information flow is allowed from low integrity subjects to high integrity subjects. Clark-Wilson model [8] attempts to capture this notion. It states that information can flow from low integrity objects to high integrity objects only through certain programs so-called transaction procedures (TP). Jaeger et al. adopted this approach and proposed a CW-lite model, where filters are deployed in all application interfaces handling information flow from low integrity data to high integrity applications [11].

There are several related approaches and tools supporting security policy analysis based on information flow control $[1,10,11,12,17]$. However, those tools and approaches cannot analyze the integrity protection of information domain, simply called domain, between services and applications. In other words, most of previous works focused on the identification of a common and minimum trusted computing base (TCB) which includes trusted subjects for an entire system. Also, they cannot analyze security of high level application domains, where TCB may have various dependable relationships with different trusted subjects. In this paper, we consider a domain as a collection of subjects and objects which jointly function for an application or system service. Hence, the trust of these application and service domains cannot be judged unless information flow between them are appropriately evaluated.

Towards high-assurance applications and services based on a minimum TCB, we propose a framework to identify policy violations caused by interactions between applications and system services. We first seek a general policy analysis method to identify the TCB of individual domains. We then build a tool to automate the analysis task and visualize policy configurations and security violations. Finally, we present general principles to resolve violations based on a domain-based Clark-Wilson security model. This paper is organized as follows. Section 2 describes some related works followed by our methodology for policy analysis in Section 3. Section 4 describes how our methodology can 
be applied to analyze SELinux policies and presents some identified security violations and solutions. Section 5 concludes this paper and presents our future work.

\section{Background and Related Work}

\subsection{SELinux Policy Related Work}

As most existing works on policy analysis focus on SELinux policy, we first give an overview here. SELinux [18] implements MAC-based mechanism in Linux kernel, where the policy is built on the Type-Enforcement model [6]. In SELinux, domains (e.g. passwd_t) are used to label processes (e.g. password management process) and types (e.g. security_t) are used to label files and other resources (e.g. files in directory / security). An SELinux policy includes a set of policy rules indicating how domain (or subject) types can access object types (e.g. allow passwd_t security_t:dir\{read search getattr\};). An access operation is specified by two pieces of information: a class (e.g. file, directory) and a permission (e.g. read, write). Although SELinux adopts RBAC to help policy organization, existing SELinux policy analysis works mainly focus on the Type-Enforcement model.

Previous methods and tools developed to analyze SELinux policies include Gokyo [11, 12], SLAT (Security Enhanced Linux Analysis Tool) [10], PAL (Policy Analysis using Logic Programming) [17], APOL [1] and SEAnalyzer [7]. Gokyo is used to check the integrity protection of the TCB in SELinux. The integrity of the TCB holds if there is no subject identified by a security type in SELinux -that can be written by a type outside the TCB and read by a type inside the TCB, except for special cases in which a designated trusted program sanitizes untrusted data when it enters the TCB. Gokyo mainly identifies a common TCB in SELinux but a typical system may have multiple security goals with obviously different kinds of trust relationships. Hence, Gokyo cannot cover all the aspects of policy violations. SLAT [10] defines an information flow model to analyze SELinux policies. In SLAT, the model defines information flow relations as flow transitions based on the write and read operations. Through a set of flow transition relationships, a path is defined to reflect a sequence of events. Sarna-Sota et al. [17] use the SLAT information flow model to implement a framework for analyzing SELinux policies, which is called PAL. PAL creates a logic program and is capable of executing queries to analyze policies. PAL is implemented using the XSB logic-programming system [3]. APOL [1] is a tool developed by Tresys Technology to analyze SELinux configuration policies. Its main features include forward and reverse domain type transition analysis, direct and transitive information flow analysis, relabel anal-
Table 1. TCB identified.

\begin{tabular}{|c|c|c|c|c|}
\hline ipsec_mgmt_t & setfiles_t & sysadm_t & initrc_t & getty_t \\
\hline load_policy_t & hwclock_t & syslogd_t & mount_t & apt_t \\
\hline admin_passwd_exec_t & automount_t & ldconfig_t & kernel_t & klogd_t \\
\hline checkpolicy_t & cardmgr_t & fsadm_t & snmpd_t & init_t \\
\hline bootloader_t & logrotate_t & newrole_t & quota_t & dpkg_t \\
\hline local_login_t & sshd_login_t & useradd_t & passwd_t & sshd_t \\
\hline
\end{tabular}

ysis, and type relationship analysis. SEAnalyzer [7] is a tool based on Colored Petri Net (CPN) to analyze SELinux policies. Policy violations can be identified through creating queries in CPN. Queries in SEAnalyzer are built on the similar information flow model with SLAT, which is mainly about TCB protection.

Although the above-mentioned tools and languages provide different ways for analyzing SELinux policies, we still need a systematic method to analyze policies effectively and seamlessly. Specifically, SLAT, PAL and APOL require an administrator to be well versed in SELinux policies to generate meaningful queries and ultimately extract meaningful information. Gokyo does not address the issue of the application/system service level integrity. SEAnalyzer tries to use CPN to aid analysis processes. However, it expresses generated policy violations in CPN token text expressions. Where the number of identified policy violations is large, especially during the initial states of a analysis process, it is still difficult for a policy administrator to understand and resolve identified violations. Our work demonstrates a new and systematic way to use CPN for automating and visualizing policy analysis.

\subsection{Trusted Computing Base}

The concept of TCB partitions hardware and software of a system into two parts: the part inside the TCB and the part outside the TCB which are referred to as trusted (TCB) and untrusted (NON-TCB) respectively. Therefore, the identification of TCB is obviously a basic problem in security policy design and management. Jeager et al. [11] attempt to identify TCB in SELinux with the following steps: (i) Domain type transition-based TCB Initial Identification to identify the initial minimum TCB based on the domain type transition relationships. The subject types such as boot_t and kernel_t can be identified as an initial TCB; (ii) Flow transition-based TCB Identification to identify other subjects that have information flow to all of the subjects in the initial TCB and to append those identified subjects to the initial TCB; and (iii) Identified TCB Adjustment to detect policy violations from NON-TCB to TCB, manually adjusting TCB based on the analysis results as shown in Table 1.

In this paper, we propose Reference Monitor based Initial TCB Identification approach for general policy analysis. Based on the definition of TCB, we identify the initial TCB through the identification of subjects functioning as the ref- 
erence monitor. Applying our main idea to SELinux, we determine the types that are responsible for the reference monitor such as checkpolicy_t and load_policy_t as part of the TCB. To support these services, we first identify other types such as kernel_t in the initial TCB. After the reference monitor based TCB identification is complete, we apply the method of flow transition and TCB adjustment to help accomplish the TCB identification task. In our work, through automatic identification process, we successfully identify several additional types including kudzu_t, lvm_t, and restorecon_t in the TCB.

\section{Our Methodology for Policy Analysis}

In this section, we present a formal specification of policy graph and information flow transitions. We then discuss our strategies to identify the security properties of a policy including different TCBs and security violations. Finally, we discuss general principles to resolve policy violations.

\subsection{Security Policy}

Our security model is similar to many traditional approaches [14]. Specifically, a set of sensitive resources (e.g., files, directories, sockets, and processes) to be protected in a system are called objects, and the active entities performing actions (rights or permissions) on objects are called subjects (e.g., users and representative processes). A security policy is composed of a set of subjects, a set of objects, and the corresponding relationships indicating whether a subject can perform what kind of actions on an object. For information flow purpose, all operations between subjects and objects can be classified as write_like or read_like and operations between subjects can be expressed as calls [10]. Figure 1 shows the information flow relations with these three types of operations between subjects and objects. Figure 1 (a) describes that if subject $x$ can write to object $y$, then there is information flow from $x$ to $y$, which is denoted as write $(x, y)$. Figure 1 (b) shows that if subject $x$ can read object $y$, then there is information flow from $y$ to $x$, which is denoted as $\operatorname{read}(y, x)$. Figure 1 (c) describes that if subject $x$ can call another subject $y$, then there is information flowing from $y$ to $x$, which is denoted as $\operatorname{call}(y, x)$. Based on these concepts, we arrive the definition of policy graph to express information flow relations between subjects, objects, and operations.

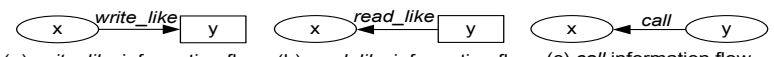

(a) write_like information flow (b) read_like information flow (c) call information flow

\section{Figure 1. Types of information flow relations.}

Definition 1 A Policy Graph of a system is a directed graph $G=(V, E)$, where a set of vertices $V$ represents all subjects and objects in the system, and a set of edges $E=V \times V$ represents all information flow relations between the subjects and objects. That is,

- $V=V_{o} \cup V_{s}$, where $V_{o}$ and $V_{s}$ are the sets of nodes that represent objects and subjects, respectively.

- $E=E_{r} \bigcup E_{w} \bigcup E_{c}$. Given a subject $x, y \in V_{s}$ and an object $o \in V_{o}$ :

- $(x, o) \in E_{w}$ if and only if write $(x, o)$.

$-(o, y) \in E_{r}$ if and only if read $(o, y)$.

$-(y, x) \in E_{c}$ if and only if $\operatorname{call}(y, x)$.

\subsection{Information Flow Transitions}

In a policy graph, if subject $s_{1}$ can write to object $o$, and $o$ can be read by another subject $s_{2}$, then it implies that there is an information flow transition from $s_{1}$ to $s_{2}$, denoted as flowtrans $\left(s_{1}, s_{2}\right)$. Also, if $s_{2}$ can call $s_{1}$, then there is the flow transition from $s_{1}$ to $s_{2}$. The sequence of flow transitions between two subjects represents an information flow path.

Definition 2 In a policy graph $G=(V, E)$, for any $s_{1}, s_{2} \in$ $V$, an information flow transition flowtrans $\left(s_{1}, s_{2}\right)$ exists if $\exists o \in V$, write $\left(s_{1}, o\right) \wedge \operatorname{read}\left(o, s_{2}\right)$, or call $\left(s_{1}, s_{2}\right)$. We also say that predicate flowtrans $\left(s_{1}, s_{2}\right)$ is true if flowtrans $\left(s_{1}, s_{2}\right)$ exists.

Definition 3 In a policy graph $G=(V, E)$, an information flow path flowpath $\left(s_{1}, s_{n}\right)$ exists and predicate flowpath $\left(s_{1}, s_{n}\right)$ is true if flowtrans $\left(s_{1}, s_{n}\right)$, or $\exists s_{i} \in$ $V$, flowpath $\left(s_{1}, s_{i}\right) \wedge$ flowpath $\left(s_{i}, s_{n}\right)$.

\subsection{TCB of a Domain}

Protecting a system's TCB can satisfy a particular security goal such as protecting kernel integrity. However, some of other security goals such as separation of different processes or limiting the privileges of certain processes cannot be satisfied at the same time. To address this, we propose a new concept called the domain TCB to determine if a policy can satisfy such kind of security goals.

Definition 4 Let $d$ be an information domain functioning as certain application or system service through a set of related subjects and objects. The TCB $(d)$ is a set of subjects in $d$ which has the same level of security sensitivity.

Superficially, a $T C B(d)$ represents a set of subjects and objects that handle or contain all critical data processed for the functionality of domain $d$. Note that the concept of information domain has much wider scope than the domain type in SELinux in this paper. For example, a web server domain running in a system consists of many subjects-such 
as processes, plugins, tools-and objects including data files, config files, and logs. We consider all of these subjects and objects as the TCB of this domain, while its network object such as socket: 80 is not considered as its TCB since it may accept low integrity data. Our objective is to identify the TCB of each domain and consider its integrity for assuring secure applications and system services. The following principles for the $\mathrm{TCB}(\mathrm{d})$ identification are proposed. For sake of clarification, the kernel-level minimum TCB we introduced in Section 2.2 is called system or global TCB.

- Keyword-based TCB $(d)$ identification: To identify the $\mathrm{TCB}(\mathrm{d})$ subjects of certain application or system service domain, we first identify all of subjects related to the integrity of the target domain based on some keywords. For example, in SELinux we use the keyword httpd to identify its initial TCB(d) subjects for the TCB of a web server, and mail and sendmail to identify its initial TCB(d) subjects for the TCB of mail service domain.

- Flow transition-based TCB $(d)$ identification: The subjects that can flow only to or from the initial identified $\mathrm{TCB}(\mathrm{d})$ are included in the domain TCB.

- Policy violation-based TCB $(d)$ adjustment: Through identifying the policy violations, we adjust the TCB(d) with incorrectly included or excluded subjects.

\subsection{Domain-based Integrity Model}

The concepts of flow transitions and paths are used in our information flow-based policy analysis for domain TCB and policy violation identifications. For this purpose, we need to identify which flow transitions and paths can cause possible violations. Specifically, as integrity protection is the focus of this paper, we identify policy violations based on an integrity model. Traditional integrity models include Biba [5] and Clark-Wilson [16]. Biba integrity property is fulfilled if a high integrity process cannot read lower integrity data, execute lower integrity programs, or obtain lower-integrity data in any other manner. Clark-Wilson provides a different view of dependencies, where low integrity data can flow to high integrity only through particular information flow channels, TPs or filters in CW-lite [11]. A typical example of filter can be a firewall, and a valid flow transition is that a mail application receives mails by calling the firewall application to sanitize data before handling.

As communication and collaboration between applications and services are frequently required in most contemporary systems, one-way information flow with Biba would be sufficient for the most cases. However, filters between high integrity and low integrity are necessary for TCB and NON-TCB isolations, but may not be flexible for domainbased isolations. For example, in SELinux, processes of user applications and staff applications are required to be isolated, which both are beyond the minimum and global system TCB. Hence, our approach is a domain-based ClarkWilson isolation model. Specifically,

Definition 5 Clark-Wilson is satisfied for an information domain $d$ only if for any information flow path in $d$, (i) all nodes along the path are in $T C B(d)$, or (ii) it flows to $T C B(d)$ from system $T C B$, or (iii) it flows to $T C B(d)$ from other domain TCBs with the legitimate filter.

Through this definition, Clark-Wilson isolation requires the system's information flow adhere either within a domain TCB from system TCB to a domain TCB, or between domain TCBs via filters. In this paper we do not discuss the integrity of filters, which can be ensured with integrity measurement and attestation mechanisms [15]. Instead, we assume that filters always correctly sanitize data between domains. In practice, filters between domains are application or service dependent. For instance, a firewall works as a filter for mail service by checking incoming mails before sending to a mail application. However, for a service like logrotate, the firewall does not work as a filter because logrotate enables automatic rotation of log files and does not depend on data in the files. In our work, initially we do not have a set of predefined filters. After detecting a possible policy violation, we try to identify a filter subject to resolve the violation.

\subsection{Violation Detection and Resolution}

Based on our domain-based Clark-Wilson model, we treat a $\mathrm{TCB}(\mathrm{d})$ as an isolated information domain. We use the following two rules for identifying possible policy violations in a system.

Rule 1 If there is an information flow path from a subject out of the system TCB to the system TCB without passing any identified filter, there is a policy violation for protecting the system TCB.

Rule 2 If there is an information flow path from $\operatorname{TCB}\left(d_{x}\right)$ to $T C B\left(d_{y}\right)$ without passing any filter, there is a policy violation for protecting $\operatorname{TCB}\left(d_{y}\right)$.

After initial policy violations are identified with these two rules, we use different strategies to resolve those violations. Specifically, for a violation, we first check if it can be solved by adding or removing related subjects to or from the domain TCB. This causes no change to the policy graph. Secondly, we check if a subject along the violated information flow path can be regarded as a filter. If a filter is identified, then the violation is a false alarm and there is no change to the policy graph. Thirdly, we attempt to modify 


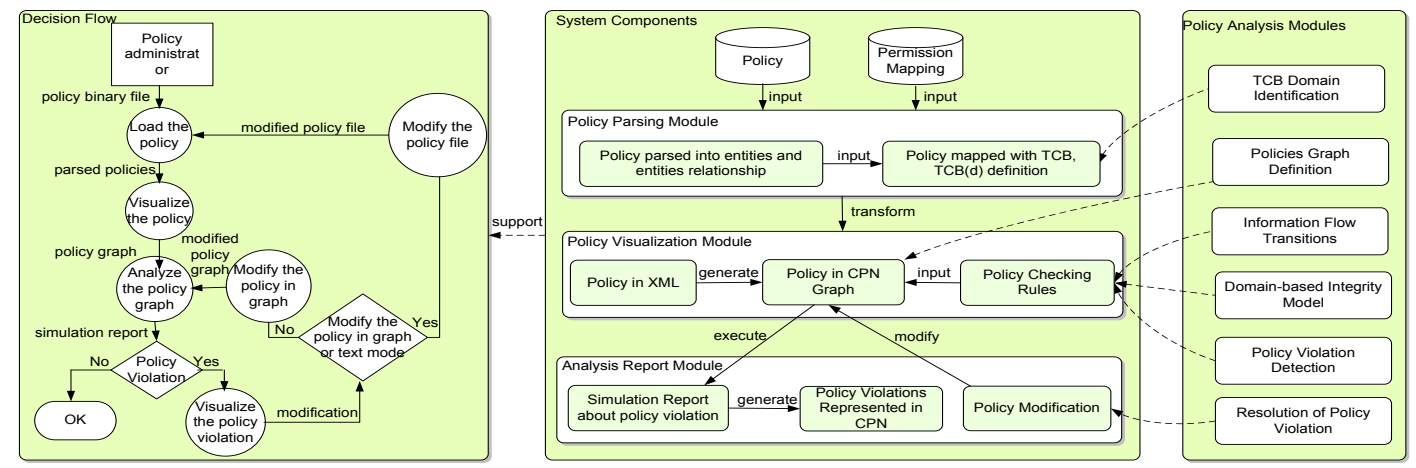

Figure 2. Policy analysis framework.

policy graph, either by excluding subjects or objects from the violated information flow path, or by replacing subjects or objects with more restricted privileges. Finally, we insert filters that act as a high-assurance gateway between unauthorized subjects and protected subjects.

\section{Realization and Experiments}

We built our prototype based on Colored Petri Nets, which is a powerful graph-based analysis tool for system modeling [13]. A Petri Nets includes three basic components: places, transitions, and arcs. Arc expressions specify a collection of tokens, which can be added to or removed from places. If a transition input place contains at least one token that is equal to the corresponding arc expression, the transition is enabled. The difference between $\mathrm{CPN}$ and Petri Nets is the inclusion of color sets in CPN, which can be viewed as abstract data types in a programming language. These types determine data attributes and operations used in arcs, guards, and initialization expression functions. CPN can support graph hierarchy, zoom in, zoom out, color expression, and so on. Also, CPN has a simulator to support execution of CPN models. The simulation is to validate whether a system works correctly reflecting the design principles. It supports both interactive simulation and automatic simulation. In the interactive simulation, a user can set breakpoints, choose between enabled binding elements, change markings of places, and study the token in detail, while the simulator makes random choices of the enabled binding elements and automatically executes the whole CPN models in the automatic simulation.

Figure 2 shows our policy analysis framework that includes Policy Analysis Modules, System Components, and Data Flow for policy analysis. Policy Analysis Modules are composed of several analysis components such as $T C B$ Domain Identification, Policy Graph Definition, Information Flow Transitions, Domain-based Integrity Model, Policy Violation Detection and Resolution of Policy Violation that are designed based on the policy analysis methodology proposed in Section 3. For the System Components, the Policy Parsing Module is to parse and map the operations between types to write_like, read_like, or call operations. For example, the operation like getattr can be mapped to a read_like operation. System TCB and domain TCB are discovered and inserted into the policies using the $T C B$ Domain Identification module in Policy Analysis Modules. Through the Policy Visualization Module, a security policy is transformed into an XML file, which is transformed into a CPN graph based on the Policies Graph Definition module. A set of policy checking rules are taken as an input into the CPN graph, and the generated CPN graph is executed with the CPN simulation function. The policy checking rules are built by using the modules in Policy Analysis Modules such as Information Flow Transitions, Domain-based Integrity Model, and Policy Violation Detection modules. The Analysis Report Module generates a simulation report containing information about policy violations by executing the policies in CPN graph and then the simulation report is transformed into another XML file. The graph along with policy violation specifications is also generated through CPN as an output. In addition, this module supports policy modification performed by Policy Violation module. The Decision Flows components govern all processes in our framework.

\subsection{Policy in XML}

Based on the definition of policy graph, we design a scheme to express policies in XML. As shown in Figure 3, the XML file generated from a parsed SELinux policy has two elements: $D T$ and $D D$. The $D T$ element includes three sets of subelements: (1) non replicated operation relationships between types, (2) the types that can flow out and into other types, and (3) the starting types which cannot have a flow into by any other types. Also, it may contain the types that can only be flew into. The $D D$ element specifies information about non-replicated transition rela- 
tionships between types.

In our experiments, we parsed a real world SELinux binary policy file policy.19 into a defined policy structure based on the source package of APOL [1] with our parsing tool. Then, we retrieved the information about subject types, object types, and related rules. Using LibXML2 [2], we transformed these information into an XML file conforming the defined policy template. Based on our TCB identification strategy mentioned in Section 2.2, we carried out the system TCB identification. The collection of the domain TCBs for all applications and system services was performed based on our $\mathrm{TCB}(\mathrm{d})$ identification method. In the example SELinux policy, there are 35 user applications, 37 staff applications, 34 administrative applications, and 84 system services. In user, staff, and sysadm levels, we identified information domains as $d_{\text {user }}$, $d_{\text {staff }}$ and $d_{\text {sysadm }}$, respectively. More fine-grained domain identifications are performed against different applications and services within these domains. For example, the user_mozilla_t related domain should be isolated from the user_xserver_t related domain. Table 2 shows the comprehensive list of TCBs that our tool successfully identified. The list of system service daemons are also shown in Table 2 and each daemon is identified as an individual domain.

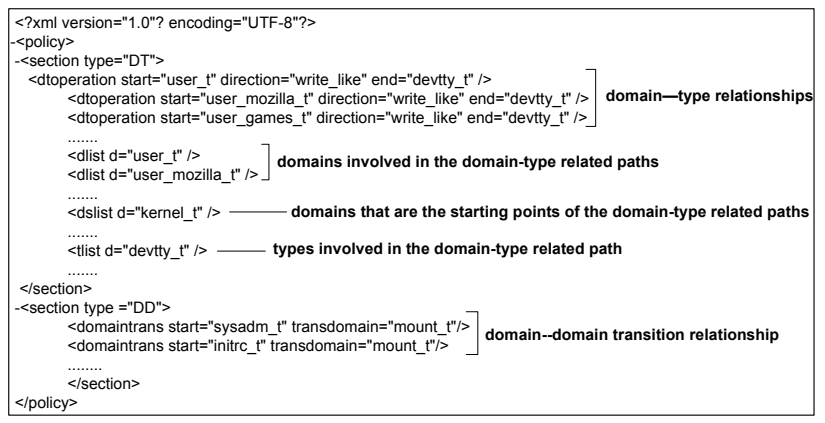

Figure 3. SELinux policy rules in XML format.

\subsection{Graph-based Policy Analysis and Vi- olation Identification}

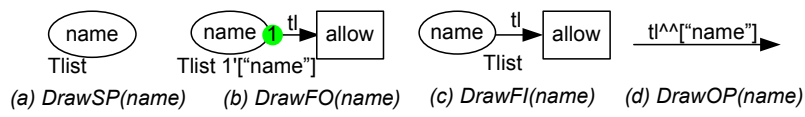

Figure 4. Graph drawing.

To automatically visualize a policy, we use Extensible Stylesheet Language (XSL) [4] to transform a policy in
Table 2. TCB(d) identified.

\begin{tabular}{|c|c|c|c|}
\hline \multicolumn{4}{|c|}{$\mathbf{T C B}\left(d_{u s e r}\right)$} \\
\hline user_crond_t & user_crontab_t & \multicolumn{2}{|c|}{ user_xserver_t } \\
\hline user_gpg_t & user_home_ssh_t & \multicolumn{2}{|c|}{ user_mozilla_dbusd_system_t } \\
\hline user_mail_t & user_screen_t & \multicolumn{2}{|c|}{ user_gpg_helper_t } \\
\hline user_tvtime_t & user_mplayer_t & \multicolumn{2}{|c|}{ user_mozilla_javaplugin_t } \\
\hline user_lpr_t & user_spamc_t & \multicolumn{2}{|c|}{ user_games_t } \\
\hline user_uml_t & user_mozilla_t & \multicolumn{2}{|c|}{ user_dbusd_user_t } \\
\hline user_ssh_t & user_gag_agent_t & \multicolumn{2}{|c|}{ user_ssh_agent_t } \\
\hline user_gph_t & user_chkpwd_t & \multicolumn{2}{|c|}{ user_ssh_keysign_t } \\
\hline user_locate_t & user_vmware_t & \multicolumn{2}{|c|}{ user_dbusd_system_t } \\
\hline user_dbusd_t & user_lockdev_t & \multicolumn{2}{|c|}{ user_spamssassin_t } \\
\hline user_irc_t & user_cdrecord_t & \multicolumn{2}{|c|}{ user_gpg_pinentry_t } \\
\hline user_xauth_t & user_mencoder_t & & \\
\hline \multicolumn{4}{|c|}{$\mathbf{T C B}\left(d_{\text {staff }}\right)$} \\
\hline staff_crontab_t & staff_xserver_t & \multicolumn{2}{|c|}{ staff_mozilla_javaplugin_t } \\
\hline staff_gpg_t & staff_mencoder_t & \multicolumn{2}{|c|}{ staff_ssh_keysign_t } \\
\hline staff_su_t & staff_cdrecord_t & \multicolumn{2}{|c|}{ staff_home_ssh_t } \\
\hline staff_ssh_t & staff_vmware_t & staff_db & isd_system_t \\
\hline staff_gph_t & staff_chkpwd_t & staff_u & serhelper_t \\
\hline staff_crond_t & staff_screen_t & staff_g & gg_helper_t \\
\hline staff_lpr_t & staff_xauth_t & staff_sp & massassin_t \\
\hline staff_locate_t & staff_lockdev_t & staff. & mplayer_t \\
\hline staff_irc_t & staff_spamc_t & staff_mozill & _dbusd_system_t \\
\hline staff_mail_t & staff_games_t & staff_es & pg_agent_t \\
\hline staff_sudo_t & staff_mozilla_t & staff_gp & g_pinentry_t \\
\hline staff_uml_t & staff_tvtime_t & staff_es & sh_agent_t \\
\hline staff_dbusd_t & & & \\
\hline & $\mathbf{T C B}\left(d_{s y s a}\right.$ & & \\
\hline sysadm_su_t & sysadm_mozilla_t & sysadm_mo & illa_javaplugin_t \\
\hline sysadm_gph_t & sysadm_spamc_t & sysadm_s & pamassassin_t \\
\hline sysadm_sudo_t & sysadm_games_t & sysadm_mozi & la_dbusd_system_t \\
\hline sysadm_uml_t & sysadm_cdrecord_t & sysadm & ssh_agent_t \\
\hline sysadm_irc_t & sysadm_crontab_t & sysadm & gpg_agent_t \\
\hline sysadm_crond_t & sysadm_passwd_t & sysadm & userhelper_t \\
\hline sysadm_gpg_t & sysadm_mplayer_t & sysadm & sh_keysign_t \\
\hline sysadm_mail_t & sysadm_xserver_t & sysadm_d & pusd_sysadm_t \\
\hline sysadm_xauth_t & sysadm_dbusd_t & sysadm_d & busd_system_t \\
\hline sysadm_ssh_t & sysadm_screen_t & sysadm & gpg_pinetry_t \\
\hline sysadm_lpr_t & sysadm_chkpwd_t & sysadm. & gpg_helper_t \\
\hline sysadm_vmware_t & & & \\
\hline & Daemon & & \\
\hline uml_switch_t & postfix_bounce_t & vpnc_t & nfsd_t \\
\hline canna_t & cyrus_t & dhcpe_t & udev_t \\
\hline ipsec_t & mdadm_t & apmd_t & ntpd_t \\
\hline ssh_keygen_t & telnetd_t & zebra_t & nscd_t \\
\hline dovecot_auth_t & slrnpull_t & saslauthd_t & howl_t \\
\hline prelink_t & tftpd_t & sendmail_t & rpm_t \\
\hline i18n_input_t & fetchmail_t & smbd_t & gpm_t \\
\hline privoxy_t & bluetooth_t & nmbd_t & lpd_t \\
\hline ypbind_t & ypserv_t & crond_t & pppd_t \\
\hline cupsd_t & mysquld_t & squid_t & mrtg_t \\
\hline dhepd_t & $\operatorname{dictd} \_t$ & arpwatch_t & hald_t \\
\hline cpuspeed_t & inetd_t & httpd_t & innd_t \\
\hline spamd_t & timidity_t & irqbalance_t & acct_t \\
\hline vmware_t & winbind_t & kadmind_t & $\mathrm{xdm} \_t$ \\
\hline networkmanager_t & dmesg_t & radiusd_t & rshd_t \\
\hline printer_t & rhgb_t & radvd_t & ptal_t \\
\hline slapd_t & gssd_t & auditd_t & rpcd_t \\
\hline fingerd_t & named_t & dovecot_t & ftpd_t \\
\hline krb5kdc_t & procmail_t & hostname_t & rdisc_t \\
\hline postgresql_t & updfstab_t & cpucontrol_t & ping_t \\
\hline games_t & iptables_t & sound_t & \\
\hline
\end{tabular}

XML to CPN graph. The following basic functions are designed for the CPN graph generation as shown in Figure 4.

- $\operatorname{DrawSP}($ name $)$ is to draw the types that no information can flow into. This function draws a place that contains the initial marking whose value is the name of the place, and the arc that has a variable to express 


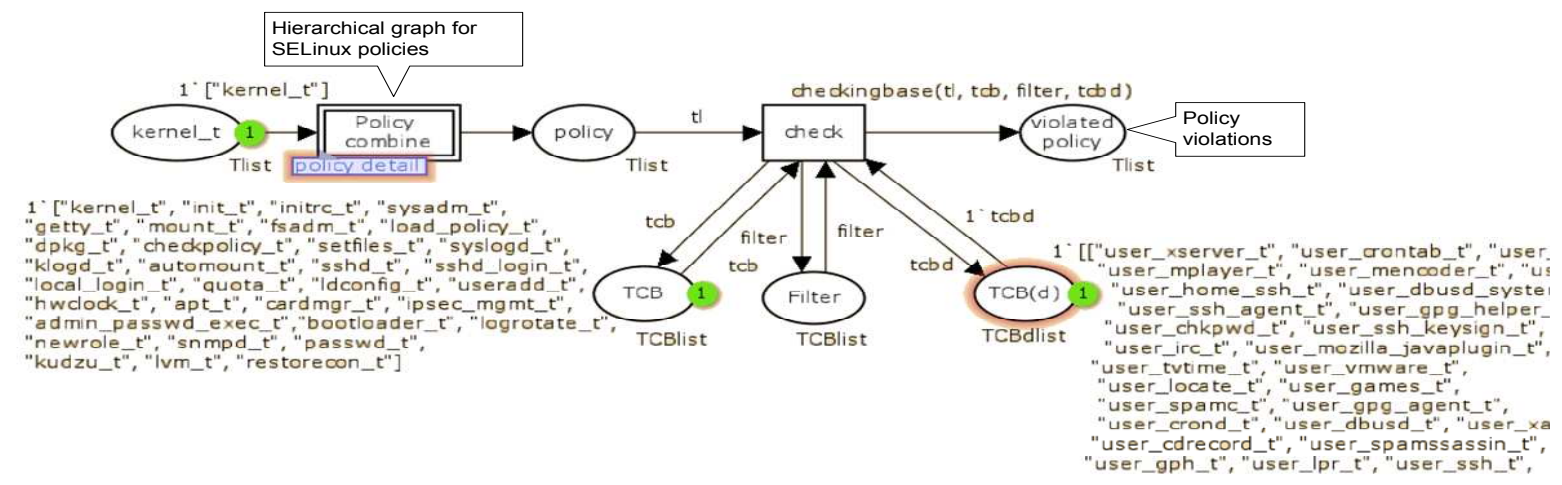

Figure 5. Policy visualization with hierarchy.

information contained in the place and the transition called allow. dlist is a color set of the place expressing a string list containing one or more type names.

- DrawFO(name) is to draw the places for types from which information can flow out. It is similar to DrawSP(name), but the places generated with this function do not have initial markings.

- DrawFI(name) is to draw places containing types from which no information can flow out.

- $\operatorname{DrawOP}($ name) is to draw an arc that connects types. The expression on the arc is to generate a list expressing flow transitions caused by domain type transitions or operations between types.

With the above functions, XML-based SELinux policy rules are parsed into a CPN graph. In order to perform the process of detecting policy violations in $\mathrm{CPN}$, the TCB related definitions, filter information and the rules for policy violation identification are also expressed in XML and transformed into CPN graphs. As stated in Section 3.4, it is hard to initially identify filters so we set initial filter values to null and put this information into the policy XML file. Later, when possible policy violations were detected, filters were identified and added to the XML file through CPN. Figure 5 illustrates a snapshot of our policy visualization.

Simulation is a technique supported by CPN to analyze a system by conducting controlled experiments [13]. In our experiments, we utilize the simulation feature to generate policy violations with text expressions. To better understand the violations, we visualize the generated expressions with another XML transformation. In our simulation, policy analysis results are stored in a simulation report. Through parsing the simulation report, we generate information about policy violation specifications and produce a new XML file with the similar XSL algorithm. The generated XML file is transformed into another CPN graph for visualization. Figure 6 shows an identified policy violation, specifying that some NON-TCB domain types can write to the type devtty_t, which can be read by some TCB types. As examples of system TCB isolation violations, Table 3 includes the identified policy violations caused by the identified information flow path from NON-TCB subjects to system TCB subjects $\mathrm{f}$ sadm_t and snmpd_t. As examples of TCB(d) isolation violations, Table 3 also shows all policy violations identified for protecting sysadm_xserver_t, which belongs to $\operatorname{TCB}\left(d_{\text {sysadm }}\right)$.

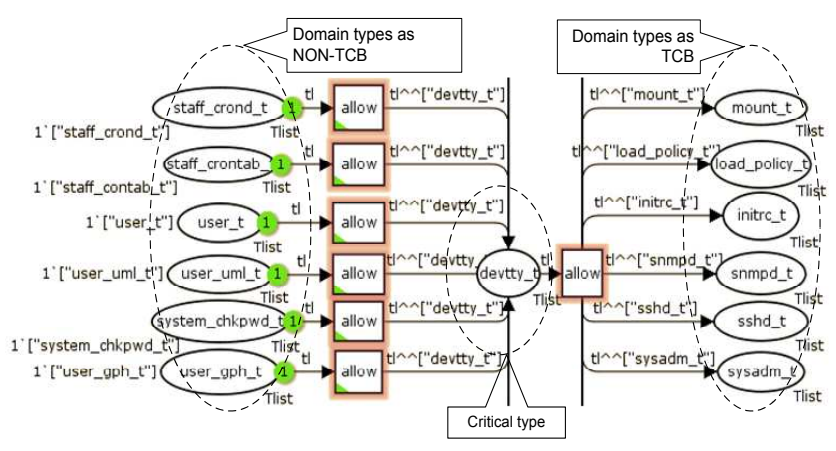

Figure 6. Example violations.

Based on the principles proposed in Section 3, our experiments also dealt with corresponding methods for resolving the policy violations. There are policy violations ignored because of its relationship to the filter. For example, the policy violations caused by writing and reading dev log_t can be ignored. devlog_t is used to label the log files of syslogd_t, which provides two system utilities: system logging and kernel message trapping. devlog_t is also used to store $\log$ information rather than the information of certain resources. Hence, even though there is an information flow from NON-TCB subject user_mozilla_t to TCB subject fsadm_t through devlog_t, it is a policy violation that can be ignored.

Most policy violations need to be resolved through policy modifications. For example, to the policy violations caused by the read and write accesses to 
Table 3. Policy violation examples.

\begin{tabular}{|c|c|c|c|}
\hline \multicolumn{5}{|c|}{ fsadm_t, snmpd_t, and mount_t related violations } \\
\hline Subject & Type:Class & Subject & Resolution \\
\hline 200 & network & fsadm_t & Filter \\
\hline 2 & mnt_t:dir & fsadm_t & Modify \\
\hline hotplug_t & etc_runtime_t:file & fsadm_t & Ignore \\
\hline 33 & unpriv_userdomain:fd use & fsadm_t & Modify \\
\hline 134 & initrc_t:fifo_file & fsadm_t & Modify \\
\hline 16 & removable_device_t:chr_file & fsadm_t & Modify \\
\hline 3 & scsi_generic_device_t:chr_file & fsadm_t & Modify \\
\hline 200 & devlog_t:sock_file & fsadm_t & Ignore \\
\hline 200 & network & snmpd_t & Filter \\
\hline 2 & mnt_t:dir & snmpd_t & Modify \\
\hline hotplug_t & etc_runtime_t:file & snmpd_t & Ignore \\
\hline 200 & devtty_t:chr_file & snmpd_t & Modify \\
\hline 134 & initrc_t:fifo_file & snmpd_t & Modify \\
\hline 104 & initrc_devpts_t:chr_file & snmpd_t & Modify \\
\hline 200 & devlog_t:sock_file & snmpd_t & Ignore \\
\hline \multicolumn{5}{|c|}{ sysadm_xserver_trelated violations } \\
\hline mta_agent_t & sysadm_tty_device_t:chr_file & sysadm_xserver_t & Modify \\
\hline sound_t & sysadm_tty_device_t:chr_file & sysadm_xserver_t & Modify \\
\hline 158 & network & sysadm_xserver_t & Filter \\
\hline 10 & zero_device_t:chr_file & sysadm_xserver_t & Modify \\
\hline 158 & devtty_t: chr_file & sysadm_xserver_t & Modify \\
\hline
\end{tabular}

devtty_t, our solution is to redefine devtty_t by introducing user_devtty_t, sysadm_devtty_t, staff_devtty_t, system_devtty_t, and daemon_devtty_t. Corresponding policy rules are also modified as follows:

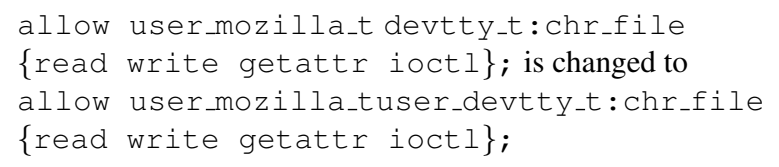

Also, the domain-based Clark-Wilson model is applied to some policy violations such as network related policy rules for violation resolution. The domain type transition relationships between the types and network filter domains need to be defined. We can assign the access of network types using network filters as follows:

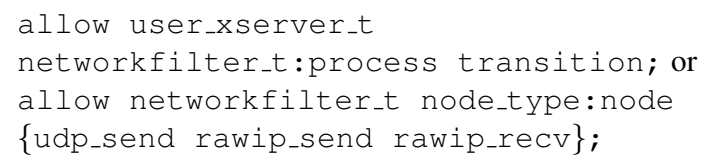

\section{Concluding Remarks}

In this paper, we have proposed a domain-based ClarkWillson model to analyze system security policies. In particular, our general method shows how we can identify system TCB and domain TCBs in the context of information domains in a system, and presents a set of rules to detect all possible policy violations from NON-TCB to system TCB, and between domain TCBs. We also automated the analysis processes using CPN and visualized graph-based violations. We adopted SELinux policy as an example to demonstrate the functionality and effectiveness of our methodology.
When one or more policy rules are modified in a policy, with our current approach the policy has to be re-loaded and a complete analysis is required to check the resolution results. Developing a fully automatic and dynamic approach for policy analysis remains as our future work, since manual analysis is still needed to identify real violations after the CPN-based analysis in our method.

\section{References}

[1] Tresys Technology APOL. Available at http://www.tresys.com/selinux/.

[2] XML Organization. The XML C parser and toolkit of Gnome. Available at http://xmlsoft.org/.

[3] XSB. Available at http://xsb.sourceforge.net/.

[4] XSL. Available at http://www.w3.org/TR/xsl/.

[5] K. J. Biba. Integrity consideratio for secure compuer system. Technical report, Mitre Corp. Report TR-3153, Bedford, Mass., 1977.

[6] W. E. Boebert and R. Y. Kain. A practical alternative to hierarchical integrity policies. In Proceedings of the Eighth National Computer Security Conference, 1985.

[7] Y.-M. Chen and Y.-W. Kao1. Information flow query and verification for security policy of security-enhanced linux using cpn. In International Workshop on Security (IWSEC), 2006.

[8] D. D. Clark and D. R. Wilson. A comparison of commercial and military computer security policies. Proceedings of the IEEE symposium on security and privacy, 1987.

[9] D. E. Denning. A lattice model of secure information flow. Commun. ACM, 19(5):236-243, May 1976.

[10] J. Guttman, A. Herzog, and J. Ramsdell. Information flow in operating systems: Eager formal methods. In Workshop on Issues in the Theory of Security (WITS), 2003.

[11] T. Jaeger, R. Sailer, and X. Zhang. Analyzing integrity protection in the selinux example policy. In Proceedings of the 12th conference on USENIX Security Symposium, 2003.

[12] T. Jaeger, X. Zhang, and A. Edwards. Policy management using access control spaces. ACM Trans. Inf. Syst. Secur., 6(3):327-364, 2003.

[13] K. Jensen. Coloured Petri nets: basic concepts, analysis methods and practical use, volume 3. Springer-Verlag New York, Inc., New York, NY, USA, 1997.

[14] B. Lampson. Protection. In 5th Princeton Symposium on Information Science and Systems, pages 437-443, 1971. Reprinted in ACM Operating Systems Review 8(1):18-24, 1974.

[15] R. Sailer, X. Zhang, T. Jaeger, and L. van Doorn. Design and implementation of a TCG-based integrity measurement architecture. In USENIX Security Symposium, 2004.

[16] R. S. Sandhu. Lattice-based access control models. IEEE Computer, vol.26(no.11):9-19, 1993.

[17] B. Sarna-Starosta and S. D. Stoller. Policy analysis for security-enhanced linux. In Proceedings of the Workshop on Issues in the Theory of Security (WITS), 2004.

[18] S. Smalley. Configuring the selinux policy. http://www.nsa.gov/SELinux/docs.html, 2003. 